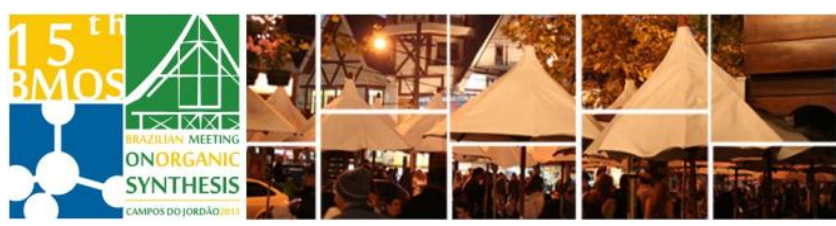

\title{
Synthesis of aromatics oximes and hydrazones with electro- withdrawing substituents as reactivators of Acetylcholinesterase
}

\author{
Petronilho, E. C. ${ }^{1}$; Pinto, A. C. ${ }^{2}$; Figueroa-Villar, J. D. ${ }^{1 *}$ \\ ${ }^{1}$ Medicinal Chemistry Group, Department of Chemistry, Military Institute of Engineering, Praça General \\ Tibúrcio, 80, 22290-270, Praia Vermelha, Rio de Janeiro, Brazil. \\ ${ }^{2}$ Institute of Chemistry, Federal University of Rio de Janeiro, Rio de Janeiro, Brazil. \\ *jdfv2009@gmail.com
}

Keywords: Acetylcholinesterase, oximes, hydrazones, Enzime reactivation.

\section{INTRODUCTION}

Neurotoxic organophosphates inhibit acetylcholinesterase (AChE) through a fosfilation process of serine involved in the hydrolysis of the neurotransmitter acetylcholine (ACh), which causes a cholinergic syndrome and eventual death from respiratory failure. There are several ways of treating intoxication by organophosphorus compounds (OPs), the AChE reactivators are oximes cationic as the pralidoxime (2-PAM) $(3),{ }^{1}$ but none of them is efficient against all the known neurotoxic agents or against all of their effects, thus demonstrating the need for development of compounds as drug candidates, being potent, selective and with a low toxicity profile to allow the treatment of poisoning caused by chemical warfare agents and pesticides. A study using molecular modeling with $a b$ initio methods suggested the evaluation of nucleophiles different from oximes, such as hydrazones. ${ }^{2}$

\section{RESULTS AND DISCUSSION}

Methylation of 2-pyridinecarboxyaldehyde (1) was accomplished by treatment with methyl iodide and the aldehyde (2) was converted to the 2-PAM (3) as shown the Figure 1.

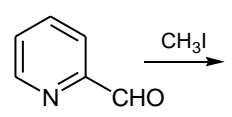

(1)

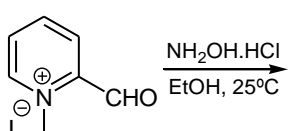

(2)

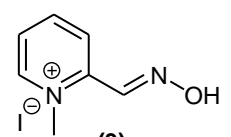

(3)
Figure 1. Synthesis of pralidoxime (3).

The synthesis of compounds analogs of 2-PAM was performed in three steps. The first step consists of an Etard oxidation using a solution of chromyl chloride in a suitable solvent, for converting the methyl group attached to the pyridine ring (4) to the aldehyde (5). In step occurs subsequent $\mathrm{N}$-alkylation using methyl iodide to obtain the cationic reactive intermediate (6). The third step consists in the use of hydroxylamine hydrochloride to form the oxime (7) and methyl hydrazine to form the hydrazone (8) desired. Synthesis of the oxime (9) hydrazone (10) were performed through using only the oxidation reaction and the hydroxylamine hydrochloride, steps 1 and 3 respectively, as shown in Figure 2.

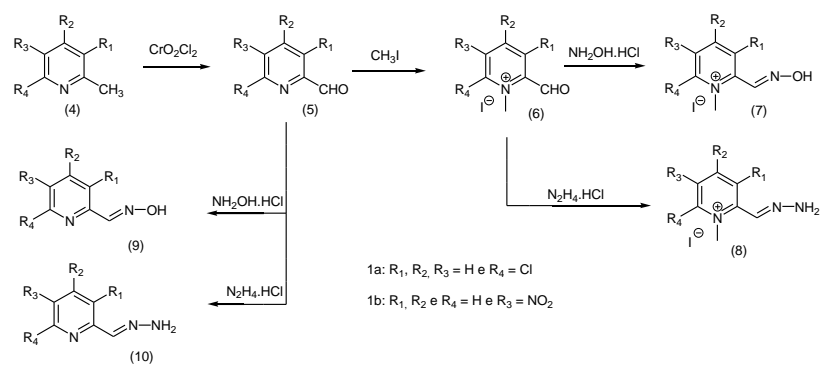

Figure 2. Synthesis of oximes and hydrazones pralidoxime analogs (2-PAM).

All compounds were characterized by FT-IR, ${ }^{13} \mathrm{C}$ NMR and ${ }^{1} \mathrm{H}-\mathrm{NMR}$.

\section{CONCLUSION}

These compounds are potential reactivators of inhibited HuAChE by organophosphorus due to the increased acidity of the hydrogens because of electro-withdrawing groups.

These are compounds will be evaluated as reactivators of Acetylcholinesterase by NMR and Ellman methods.

\section{ACKNOWLEDGEMENTS}

CAPES/Pró-defesa, CNPQ and INBEB.

\section{REFERENCES}

${ }^{1}$ Delfino, R. T., Ribeiro, T. S. \& Figueroa-Villar J. D. J. Braz. Chem. Soc., 2009, 20: 407-428.

${ }^{2}$ Delfino, R. T. \& Figueroa-Villar, J. D. J. Phys.Chem.B. 2009, 113: 8402-8411. 